\title{
Histological Evaluation Of Articular Cartilage Damage Following Radiofrequency Chondroplasty
}

\author{
Vidhya Lakshmi, Rufus V Raj, and Santosh Sahanand
}

\begin{abstract}
Background: Radiofrequency probes have been used extensively in various arthroscopic procedures. Measurable cartilage damage occurs after radiofrequency probe application. However, the histological changes of cartilage damage following radiofrequency chondroplasty have not been extensively studied.

Materials \& Methods: Articular cartilage specimens were obtained from patients undergoing total knee replacement surgeries. Radiofrequency probes were applied at 1, 3 and 5 seconds and samples were sent to histopathology laboratory. The histological changes of cartilage damage were analyzed using the International Cartilage Repair Society (ICRS) assessment scale. Safranin $O$ staining was done to assess the proteoglycan content.
\end{abstract}

Results: Histologically measurable articular cartilage damage occurs after radiofrequency probes

Conclusions: The time of application of radiofrequency probes should be optimized so as to minimize cartilage damage.

Index Terms - Articular cartilage, damage, Radiofrequency Chondroplasty,

\section{INTRODUCTION}

In Chondroplasty, Radiofrequency energy (RFE) creates a smooth cartilage surface at the expense of collagen denaturation $\&$ dose dependent chondrocyte death especially in the superficial layer. Despite the popularity and promising short term outcomes, histological damage to articular cartilage after Radiofrequency (RF) technology has not yet been extensively studied. The safety profile of RF energy and long term prognosis after treatment with this technology remains a concern for both surgeons and patients. The proposed hypothesis is that at minimal settings, radiofrequency probes cause cell death in measurable areas when applied to human articular cartilage. This article aims at studying the degree of articular cartilage damage using radiofrequency chondroplasty in humans by histological evaluation.

\section{Materials And Methods}

A controlled laboratory study was performed on articular cartilage specimen samples that were obtained from patients undergoing total knee replacement surgeries after obtaining due informed consent. The study was carried out after

Published on February 13, 2020.

Vidhya Lakshmi, PSG Institute of Medical Sciences and Research, Coimbatore, India

(e-mail: dr.vidhyalakshmi07@gmail.com)

Rufus V. Raj, Dr. Rela Institute and Medical Centre, Chennai, India

(e-mail: rufusvraj@gmail.com)

Santosh Sahanand, Ortho One hospital, Coimbatore, India.

(e-mail: sahanand@gmail.com) clearance from Institutional Ethics Committee.

Cartilage was classified into 4 grades:

- Grade I - softening of the cartilage

- Grade II - partial-thickness fibrillation and fragmentation of the cartilage

- Grades III and IV - extensive fibrillation down to the bone with much of the cartilage worn away.

Only fresh, healthy appearing Grade $\leq$ II cartilage from the distal posterior femoral condyle was included in this study. To simulate an arthroscopic procedure, samples were placed in normal saline maintained at $37^{\circ} \mathrm{C}$ in a large metal container. RF probe placed on the cartilage at 1, 3 and 5 seconds at different places.

A total of 90 samples of cartilage ( 30 cases $-1,3$ and 5 seconds after radiofrequency probes) were received in the department of Pathology. Representative bits were taken from these samples and were subjected to decalcification using nitric acid for 1 day(short DC). The specimens were processed using Leica automatic tissue processor and 3 micron sections were cut using the microtome. The slides were stained using routine Hematoxylin and Eosin stains. All these sections were also stained with Safranin O.

Safranin $O$ is a basic dye that stains growth plate cartilage and articular cartilage (proteoglycans, chondrocytes and type II collagen) in varying shades of red. The intensity of Safranin $O$ stain is proportional to the proteoglycan content in the cartilage tissue. Fast green was used for counter staining to provide contrast. Full thickness, diffuse strong staining is optimal and considered as 100\%.

International Cartilage Repair Society (ICRS II) developed a scoring system to evaluate the quality of the repair tissue. ICRS visual assessment scale assesses 6 components - matrix, cell distribution, Subchondral bone \& surface, cell population viability and cartilage mineralization.

Overall assessment - Fibrous tissue is indicative of poor quality of the repaired tissue. Articular cartilage is indicative of ideal repair/ regeneration. Fibro cartilage should be graded in the midrange of the scale (average).

\section{RESUlTS}

\section{A. Histological Assessment Of Human Cartilage Repair}

Histological assessment may provide an indication of the quality of structural repair because the organization of the tissue as well as of the components present is being evaluated. Articular cartilage is a tissue with specific organization for low friction, efficient load bearing and distribution. The major constituents are chondrocytes embedded in an extra cellular matrix along with collagen fibers and proteoglycans. Changes in structure and 
composition of cartilage lead to abnormal function. The lack of blood vessels and lymphatic supply and the presence of extra cellular matrix restrict the natural repair of cartilage. There is a clear need for a reliable, sensitive, objective and reproducible method to assess the quality of repair tissue.

In 2003, International Cartilage Repair Society (ICRS II) developed a scoring system to evaluate the quality of the repair tissue. ICRS visual assessment scale assesses 6 components - matrix, cell distribution, Subchondral bone \& surface, cell population viability and cartilage mineralization.

The following 14 parameters were analyzed based on the scoring criteria proposed by ICRS

1. Tissue morphology - hyaline, fibrous or mixed. Hyaline is glass like without any collagen fibers and hence indicates good quality repair. Fibrous indicates presence of collagen fibers which means poor healing.

2. Matrix staining - the metachromasia indicates the proteoglycan content, i.e. load bearing qualities of the cartilage. Absence of metachromatic staining using Safranin $\mathrm{O}$ is considered poor. Full thickness diffuse strong staining is optimal

3. Cell morphology - cells associated with hyaline cartilage are oval with pericellular lacuna. Cells associated with fibro cartilage are elongated and spindle shaped.

4. Chondrocytes clustering - chondrocyte cluster is defined as a group of at least 4 cells. Presence of chondrocyte clustering may indicate remodeling of cartilage during healing.

5. Surface architecture - Smooth surface is indicative of ideal repair/ regeneration. Delamination, loosening and disruptions indicate poor repair.

6. Basal Integration - Integration to subjacent bone is an important feature of cartilage repair.

7. Formation of tide mark - "Tidemark" separates the lower radial zone of cartilage from the underlying zone of calcified cartilage. A single tidemark is a feature of normal healthy cartilage The lack of a visible interface between radial zone and calcified cartilage indicates poor quality

8. Subchondral bone abnormalities/marrow fibrosis presence of an advanced/irregular Subchondral plate with or without infiltration by fibroblasts/ vascularity (marrow fibrosis) into the subchondral bone marrow is considered as poor tissue repair. Abnormal subchondral bone interface is seen in fractures, sclerosis, disruption and intense remodeling causing fibro vascular infiltration into the marrow space.

9. Inflammation - presence of lymphocytes or plasma cells in repaired cartilage is a negative feature.

10. Abnormal calcification/ ossification within the cartilage repair tissue - indicative of functional impairment

11. Vascularization (within the repair tissue) - No blood vessels in normal articular cartilage. Vascularization seen in fibrous tissue is considered as a negative feature in cartilage repair

12. Surface/ superficial assessment - upper 3rd of cartilage tissue should be intact with elongated cells and collagen fibers parallel to the surface in the most superficial zone. Severely disrupted cartilage with no obvious collagen fiber organization suggest poor healing

13. Mid/Deep zone assessment - lower $2 / 3$ rd of tissue
- hyaline cartilage should be rich in proteoglycans with no detectable collagen fibers.

14. Overall assessment - Fibrous tissue is indicative of poor quality of the repaired tissue. Articular cartilage is indicative of ideal repair/ regeneration. Fibro cartilage should be graded in the midrange of the scale (average).

15. Safranin O staining - full thickness, diffuse strong staining is optimal and considered as $100 \%$.

These histological parameters and Safranin O staining (intensity and percentage of cells) after radiofrequency probe application for 1, 3 and 5 seconds in the 30 cartilage samples are given below in Table I.

TABLE I: HISTOLOGICAL PARAMETERS AND SAFRANIN O STAINING AFTER RADIOFREQUENCY PROBE APPLICATION

\begin{tabular}{|c|c|c|c|}
\hline Parameter & $\begin{array}{l}\text { A } \\
\text { RF-1sec }\end{array}$ & $\begin{array}{l}\text { B } \\
\text { RF-3secs }\end{array}$ & $\begin{array}{l}\text { C } \\
\text { RF- 5secs }\end{array}$ \\
\hline Fibrosis of cartilage & $2 / 30$ & $4 / 30$ & $13 / 30$ \\
\hline Chondrocyte clustering & $0 / 30$ & $7 / 30$ & $16 / 30$ \\
\hline Basal integration & $18 / 30$ & $11 / 30$ & $7 / 30$ \\
\hline Lack of interface & $2 / 30$ & $12 / 30$ & $12 / 30$ \\
\hline $\begin{array}{l}\text { Abnormal } \\
\text { calcification/vascularity }\end{array}$ & $0 / 30$ & $2 / 30$ & $6 / 30$ \\
\hline Safranin $\mathrm{O}$ staining $<50 \%$ & $2 / 30$ & $5 / 30$ & $20 / 30$ \\
\hline
\end{tabular}

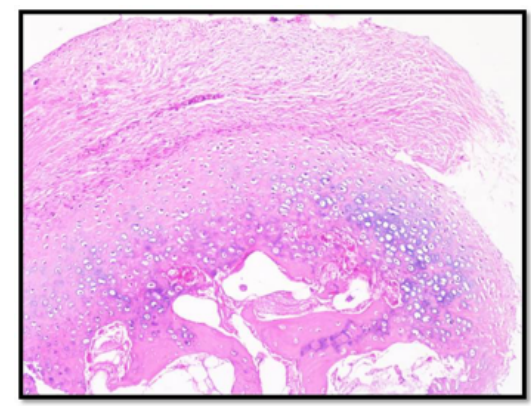

Fig. 1. Normal cartilage (H\&E 20x)

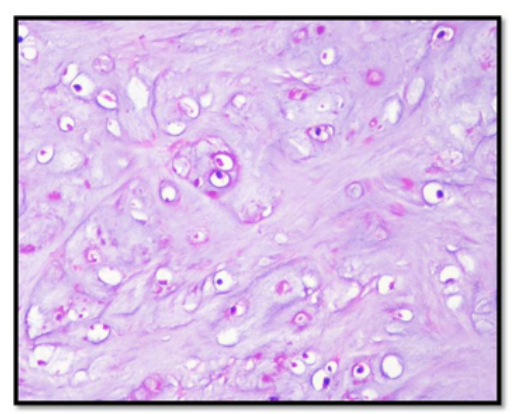

Fig. 2. Fibro-hyaline cartilage (H\&E 40x) 


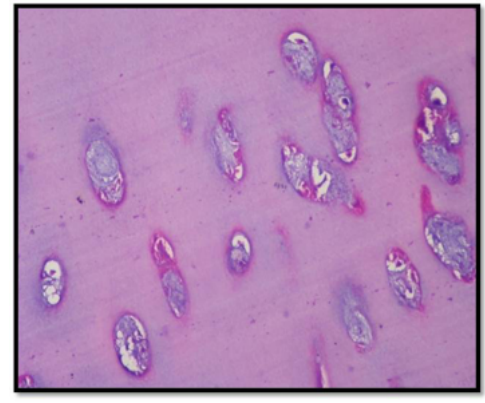

Fig. 3. Fibrous cartilage(H\&E 20x)

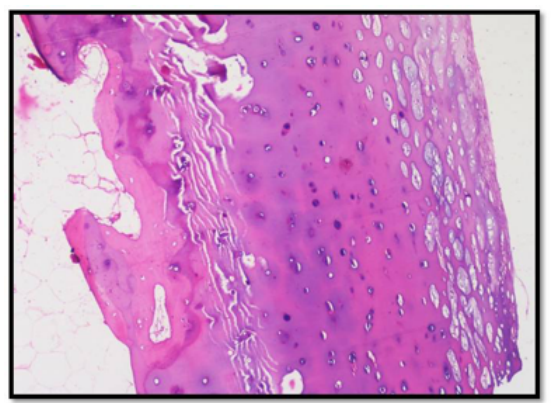

Fig. 4. Lack of interface between radial zone and calcified cartilage (H \& E $20 \mathrm{x})$

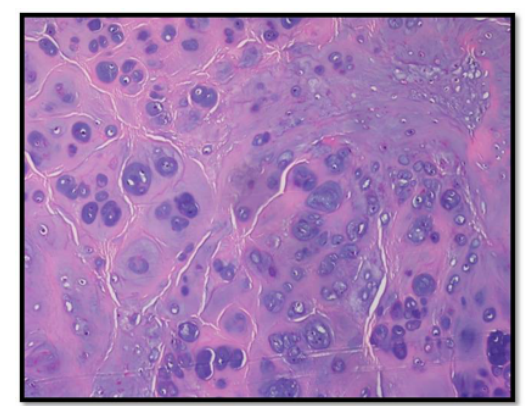

Fig. 5. Clustering of chondrocytes. (H\&E 40x)

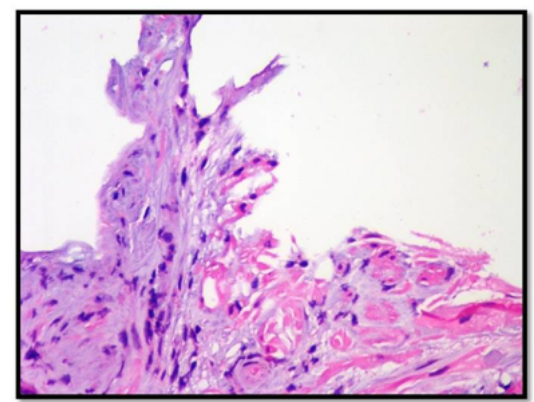

Fig. 6. Increased infiltration by fibroblasts within the marrow space. (H \& E $20 x$ )

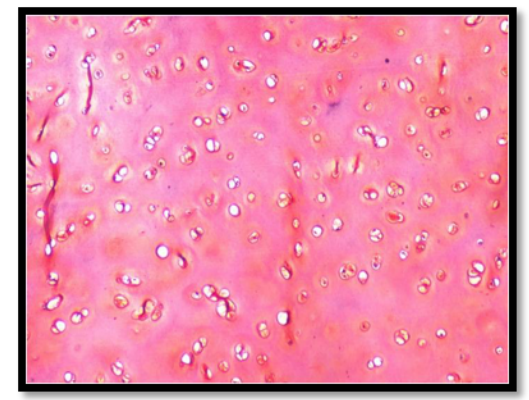

Fig. 7. Safranin O staining $(100 \%), 40 \mathrm{x}$

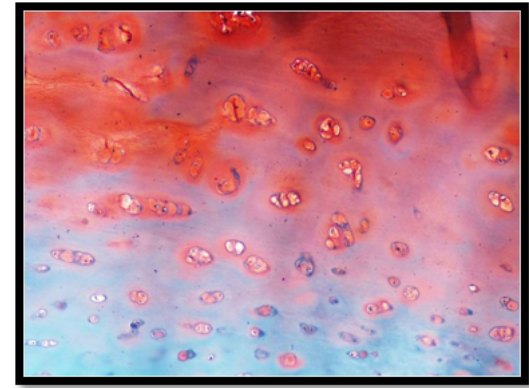

Fig. 8. Safranin O staining $(50 \%), 40 \mathrm{x}$

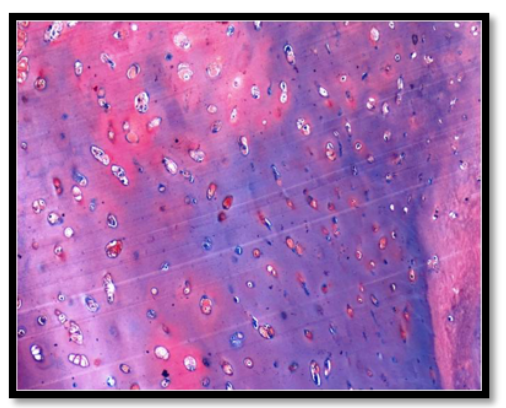

Fig. 9. Safranin O staining (25\%), 40x

\section{DISCUSSION}

Radiofrequency probe is increasingly used for arthroscopic procedures. The harmful effects of this probe on cartilage need to be studied to avoid any iatrogenic cartilage damage. Intact articular cartilage provides a smooth, lubricated gliding surface that distributes the loads of articulation in a joint. Injury and disease can alter the mechanical and biochemical properties of articular cartilage. Cartilage injury response varies depending on the extent and type of injury. The assessment of cartilage damage was based only on clinical response of the patients in earlier studies. The present study is unique in that the histological parameters of the cartilage damage were assessed using a scoring system and Safranin $\mathrm{O}$ staining of proteoglycan content. The damage to articular cartilage in the form of chondrocyte clustering, lack of interface and abnormal calcification increases with increasing time of application of $\mathrm{RF}$ probes. Also there is decrease in Safranin O positive proteoglycan content as time of probe application increases.

\section{CONCLUSION}

Histologically measurable articular cartilage damage occurs after radiofrequency probes. Considering the effects of iatrogenic damage to the cartilage, the time of application of RF probes has to be optimized.

\section{ACKNOWLEDGMENT}

The technical, theatre and nursing staff of orthopedics and pathology departments.

\section{REFERENCES}

[1] Turner AS, Tippett JW, Powers BE, Dewell RD, Mallinckrodt CH. Radiofrequency (electrosurgical) ablation of articular cartilage: a study in sheep. Arthroscopy. 1998;14:585-91

[2] Lu Y, Edwards RB, Nho S, Heiner JP, Cole BJ, Markel MD. Thermal chondroplasty with bipolar and monopolar radiofrequency energy: 
effect of treatment time on chondrocyte death and surface contouring. Arthroscopy. 2002; 18:779-88.

[3] Kaplan L, Uribe JW. The acute effects of radiofrequency energy in articular cartilage: an in vitro study. Arthroscopy. 2000; 16:2-5.

[4] Edwards RB, Lu Y, Nho S, Cole BJ, Markel MD. Thermal chondroplasty of chondromalacic human cartilage: an ex vivo comparison of bipolar and monopolar radiofrequency devices. Am J Sports Med.2002;30:90-97

[5] Lu Y, Edwards RB, Kalscheur VL, Nho S, Cole BJ, Markel MD. Effect of bipolar radiofrequency energy on human articular cartilage: comparison of confocal laser microscopy and light microscopy. Arthroscopy. 2001;17:117-23

[6] Stein DT, Ricciardi CA, Viehe T. The effectiveness of the use of electrocautery with chondroplasty in treating chondromalacic lesions: a randomized prospective study. Arthroscopy. 2002;18:190-93.

[7] Owens BD, Stickles BJ, Balikian P, Busconi BD. Prospective analysis of radiofrequency versus mechanical debridement of isolated patellar chondral lesions. Arthroscopy. 2002; 18:151-55.

[8] Lawrence Rosenberg. Chemical basis for the Histological use of Safranin O in study of articular cartilage. J Bone Joint Surg Am, 1971 Jan; 53 (1): 69 -82.

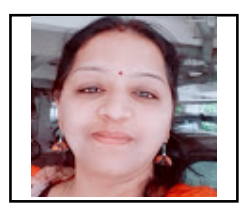

Vidhya Lakshmi S. , Date of birth - 1.4.1977; Employed as ASSOCIATE PROFESSOR, Department of pathology, PSG Institute of Medical Sciences and Research, Coimbatore, India.

Educational qualification- MD Pathology, PDCC Oncopathology.

She has been working as a full time pathologist at PSG Institute of Medical Sciences and Research since 2009. Her main areas of interests are neuropathology and Oncopathology.

Vidhya Lakshmi S is a life member of the Indian Association of pathologists and Microbiologist.

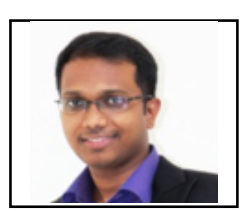

Dr. Rufus V Raj, Senior Consultant -Orthopedics, Dr.Rela Institute and Medical centre, Chennai. DNB Orthopedics, Fellowship in joint replacement, arthroscopy and sports medicine.

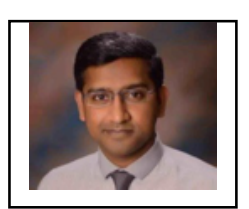

Dr. Santhosh Sahanand, Medical Superindent, Ortho One hospital, Coimbatore.MS Orthopedics, Fellowship in knee surgery, sports medicine, orthopedic rehabilitation and cartilage repair society 